\title{
Inhomogeneous STLS theory and TDCDFT
}

\author{
John F. Dobson \\ School of Biomolecular and Physical Sciences and \\ Nanoscale Science and Technology Centre \\ Griffith University, Nathan, \\ Queensland 411, Australia
}

(Dated: March 23, 2009)

\begin{abstract}
We consider the Inhomogeneous Singwi-Tosi-Land-Sjolander theory (ISTLS) of Dobson, Wang and Gould (Phys. Rev. B 66, 081108(R) (2002)), and possible generalizations, in the context of Time Dependent Current Density Functional Theory (TDCDFT). We show that ISTLS corresponds to an exchange correlation kernel $f_{x c}$ that has only a trivial frequency dependence, and a only a very simple tensorial structure. We propose approaches that may obtain more realistic structure while retaining the positive ISTLS features, namely that strong spatial inhomogeneity is allowed, with a kernel that contains physics specific to the inhomogeneous system under study, rather than being based on uniform-gas data.
\end{abstract}


hole normalization", i.e.

$$
\int f_{\lambda}^{(2)}\left(\vec{r}, \vec{p} ; \vec{r}^{\prime}, \vec{p}^{\prime}, t\right) d \vec{r}^{\prime} d \vec{p}^{\prime} \neq f(\vec{r}, \vec{p}, t)
$$

though from the definition

$$
g_{\lambda}\left(\vec{r}, \vec{r}^{\prime}\right)=\frac{n_{0 \lambda}^{2 e q u}\left(\vec{r} ; \vec{r}^{\prime}\right)}{n_{0}(\vec{r}) n_{0}\left(\vec{r}^{\prime}\right)}=\frac{\int f_{0 \lambda}^{(2)}\left(\vec{r}, \vec{p} ; \vec{r}^{\prime}, \vec{p}^{\prime}, t\right) d \vec{p} d \vec{p}^{\prime}}{\int f(\vec{r}, \vec{p}) d \vec{p} \int f\left(\vec{r}^{\prime}, \vec{p}^{\prime}\right) d \vec{p}^{\prime}} .
$$

it follows that normalization is achieved in the momentum-integrated sense for the equilibrium (time-independent) case. Notice that even in the uniform-gas version of STLS it seems there is no guarantee that the LHS and RHS of (2) are equal. There seems to be little if any discussion of this point in the literature. Indeed the factorization Ansatz (1) is used in the original STLS scheme for uniform gases [1], in the "quantum" uniform-gas generalizations of it [2], and in its generalization to inhomogeneous systems [3]. It should be stressed that this dynamic hole normalization issue does not affect the correct normalization of the static xc hole embodied in the $g\left(\vec{r}, \vec{r}^{\prime}\right)$ function derived via the fluctuationdissipation theorem from the dynamic response function $\chi$, during the STLS selfconsistency loop (Eq (6)). This is because both the KS response $\chi_{K S} \equiv \chi_{\lambda=0}$ and and the interacting response $\chi_{\lambda}=\chi_{K S} *\left(I+\chi_{K S} *\left(V_{\lambda}^{\text {coul }}+f_{x c}\right) * \chi_{\lambda}\right)$ satisfy particle conservation in the form $\int \chi\left(\vec{r}, \vec{r}^{\prime}, \omega\right) d \vec{r}=0$ regardless of the value of $f_{x c}$. (To see that this implies static xc hole normalization, consult (e.g.) [4].)

Despite the crudeness of the factorization scheme (1), the STLS formalism gives excellent correlation energies for both 3D and 2D homogeneous electron gases, up to relatively large values of the inter-electron spacing parameter $r_{s}$ (within $<2 \%$ of the $3 \mathrm{D}$ diffusion Monte Carlo results [5] at metallic densities, and still only about $5 \%$ off at $r_{s}=40$, a significantly correlated case). As a result of the crude factorization (1), the STLS scheme has a number of shortcomings including unphysical negative values of the on-top pair factor $g(\vec{r}, \vec{r})$ and failure to satisfy the compressibility sum rule. The failure of the STLS g-factor at small separations is fortunately not very influential in prediction of correlation energies, because of the relatively "soft" character of the Coulomb pair potential at short range. This would clearly be much more of a problem (e.g.) in many-nucleon systems with a hard-cored potential. Further work addressing these difficulties [2, 6-9] did not, however, significantly improve the predicted uniform egas correlation energy. Therefore in the ISTLS approach [3], the focus was on generalizing the original semi-classical STLS scheme [1] to inhomogeneous systems. 
Aside from the groundstate energy, the STLS scheme also produces detailed spectral information as contained in the density-density response function $\chi\left(\vec{r}, \vec{r}^{\prime}, \omega\right)$. This information has been used, for example, to look at the softening of plasmons at large $r_{s}$ in uniform systems. However the STLS spectral information has not had the same confirmation from Monte Carlo data that the STLS groundstate energy $E^{g r}$ has enjoyed. Since $E^{g r}$ is a frequency and spatial integral over $\chi$, it is quite likely that the STLS spectral data is "good" only in some frequency-averaged sense. Indeed we will show below that the STLS scheme, suitably generalized ([3]," ISTLS") to spatially inhomogeneous situations, corresponds to a tensor exchange-correlation kernel $\mathbf{f}_{x c}\left(\vec{r}, \vec{r}^{\prime}, \omega\right)$ within linear Time Dependent Current Density Functional Theory (TDCDFT, [10]) that is only trivially dependent on frequency, and has a relatively trivial tensor character. On the other hand, this $\mathbf{f}_{x c}^{I S T L S}\left(\vec{r}, \vec{r}^{\prime}, \omega\right)$ kernel has a highly non-trivial, nonlocal spatial dependence that is selfconsistently "tailored" to the particular inhomogeneous system, and is not "borrowed" from the homogeneous electron gas. Thus ISTLS goes a long way beyond the usual local density approximation for $\mathbf{f}_{x c}\left(\vec{r}, \vec{r}^{\prime}, \omega\right)$.

Correspondingly, a principal goal of the present work is to propose that it may be possible to generate a nontrivial tensor character and/or frequency dependence (i.e. non-trivial memory behavior) for $\mathbf{f}_{x c}\left(\vec{r}, \vec{r}^{\prime}, \omega\right)$ within an ISTLS-like scheme. This would then lead to a nontrivial, self-consistent nolocality of $\mathbf{f}$, both in space and in time.

In section II we first briefly recapitulate the ISTLS scheme [3]. Then in section III we make a very small extension that permits us to exhibit the specific form of the $\mathbf{f}_{x c}^{I S T L S}\left(\vec{r} \cdot \vec{r}^{\prime}, \omega\right)$ kernel of TDCDFT. In sections IV and V we compare the ISTLS kernel with those from some other theories. This brings up some formal difficulties with kernels of the STLS type. Nevertheless in sections VI and VII we propose exploration of some further generalizations of ISTLS in order to achieve an exchange-correlation kernel with non-trivial tensor character (corresponding to velocity shear effects) and non-trivial frequency dependence. The embedding of these properties in an STLS-like scheme would have the advantage of being at least plausible for systems with very strong spatial inhomogeneity, with xc properties generated, at least in part, selfconsistently for the particular system, rather than being "borrowed" from the homogeneous gas. Finally in section VIII we summarize the presentation. 


\section{ISTLS SCHEME}

As in [3] we use the constant-density adiabatic Connection - Fluctuation -Dissipation theorem approach to relate the equilibrium / groundstate properties and dynamic response of a general inhomogeneous many-electron system. Because of the application to the groundstate energy using the adiabatic connection formula, in this theory we work with a " $\lambda$-system" in which the coulomb interaction is replaced by $\lambda e^{2} / r_{12}$ and a fictitious external potential $\Delta V_{\lambda}$ is introduced to make the equilibrium density equal to the true $(\lambda=1)$ density $n_{0}(\vec{r})$ independent of $\lambda$. The causal density-density response function $\chi_{\lambda}$ is defined such that the electronic number density perturbation of the $\lambda$-system, due to a small external potential energy perturbation, is

$$
\delta n(\vec{r}, t)=\int \chi_{\lambda}\left(\vec{r}, \vec{r}^{\prime}, t-t^{\prime}\right) \delta V^{e x t}\left(\vec{r}^{\prime}, t^{\prime}\right) d \vec{r}^{\prime} d t^{\prime}+O\left(\delta\left(V^{e x t}\right)^{2}\right) .
$$

The equilibrium pair distribution $n_{2 \lambda}$ in (3) can be related to $\chi_{\lambda}$ via the fluctuationdissipation theorem, which for the $T=0 K$ application that we ultimately envisage is:

$$
n_{2 \lambda}\left(\vec{r}, \vec{r}^{\prime}\right)=n(\vec{r}) n\left(\vec{r}^{\prime}\right)-\frac{\hbar}{\pi} \int_{0}^{\infty} \chi_{\lambda}\left(\vec{r}, \vec{r}^{\prime}, \omega=i u\right) d u-n(\vec{r}) \delta\left(\vec{r}-\vec{r}^{\prime}\right) .
$$

The equilibrium pair correlation factor $g_{\lambda}\left(\vec{r}, \vec{r}^{\prime}\right) \equiv n_{2 \lambda}\left(\vec{r}, \vec{r}^{\prime}\right) /\left[\left(n(\vec{r}) n\left(\vec{r}^{\prime}\right)\right]\right.$ of the $\lambda$-system is then

$$
g_{\lambda}\left(\vec{r}, \vec{r}^{\prime}\right)=1-\frac{1}{n(\vec{r}) n\left(\vec{r}^{\prime}\right)} \frac{\hbar}{\pi} \int_{0}^{\infty} \chi_{\lambda}\left(\vec{r}, \vec{r}^{\prime}, \omega=i u\right) d u-n(\vec{r})^{-1} \delta\left(\vec{r}-\vec{r}^{\prime}\right) .
$$

The Kohn-Sham density-density response $\chi_{K S}[11]$ is defined to be that of independent electrons moving in the KS potential $v_{K S}$. Note that

$$
\chi_{K S, \lambda} \equiv \chi_{K S} \equiv \chi_{\lambda=0} .
$$

The philosophy used in [3] was to insert the semiclassical kinetic theory approach of the original STLS paper into the above formalism for inhomogeneous groundstate and response properties. The exact time evolution equation for the one-particle classical distribution $f$ is known as the first BBGKY hierarchy equation. It links $f$ to its two-body counterpart $f_{\lambda}^{(2)}\left(\vec{r}, \vec{p}, \vec{r}^{\prime}, \vec{p}^{\prime}, t\right)$ as follows [12]: 


$$
\left(\frac{\partial}{\partial t}+m^{-1} \vec{p} \cdot \frac{\partial}{\partial \vec{r}}+\vec{F}_{\lambda}^{e x t}(\vec{r}, t) \cdot \frac{\partial}{\partial \vec{p}}\right) f(\vec{r}, \vec{p}, t)=\int\left(\frac{\partial}{\partial \vec{r}} \frac{\lambda e^{2}}{\left|\vec{r}-\vec{r}^{\prime}\right|}\right) \cdot \frac{\partial}{\partial \vec{p}} f_{\lambda}^{(2)}\left(\vec{r}, \vec{p} ; \vec{r}^{\prime}, \vec{p}^{\prime}, t\right) d \vec{r}^{\prime} d \vec{p}^{\prime} .
$$

With the factorization (1) followed by linearization about the equilibrium distribution, $f=$ $f_{0}(\vec{r}, \vec{p})+\delta f(\vec{r}, \vec{p}, t)$, we obtain from (8) a closed one-body kinetic equation

$$
\left(\frac{\partial}{\partial t}+m^{-1} \vec{p} \cdot \frac{\partial}{\partial \vec{r}}+\vec{F}^{(0)}(\vec{r}) \cdot \frac{\partial}{\partial \vec{p}}\right) \delta f(\vec{r}, \vec{p}, t)=-\delta \vec{F}^{e f f}(\vec{r}, t) \cdot \frac{\partial f_{0}(\vec{r}, \vec{p})}{\partial \vec{p}} .
$$

Here

$$
\begin{aligned}
\vec{F}^{(0)}(\vec{r}) & =\vec{F}_{\lambda}^{e x t}+\vec{F}_{\lambda}^{H x c 0}=\vec{F}_{\lambda}^{e x t}(\vec{r})-\int\left(\frac{\partial}{\partial \vec{r}} \frac{\lambda e^{2}}{\left|\vec{r}-\vec{r}^{\prime}\right|}\right) g_{\lambda}\left(\vec{r}, \vec{r}^{\prime}\right) n_{0}\left(\vec{r}^{\prime}\right) d \vec{r}^{\prime}, \\
\delta \vec{F}^{e f f}(\vec{r}, t) & =\delta \vec{F}^{e x t}(r, t)+\int \vec{W}_{\lambda}\left(\vec{r}, \vec{r}^{\prime}\right) \delta n\left(\vec{r}^{\prime}, t\right) d \vec{r}^{\prime}, \\
\vec{W}_{\lambda}\left(\vec{r}, \vec{r}^{\prime}\right) & =g_{\lambda}\left(\vec{r}, \vec{r}^{\prime}\right) \frac{\partial}{\partial \vec{r}} \frac{\lambda e^{2}}{\left|\vec{r}-\vec{r}^{\prime}\right|}, \delta n(\vec{r}, t)=\int \delta f(\vec{r}, \vec{p}, t) d \vec{p}
\end{aligned}
$$

Because (9) is linear and time invariant, its solution can be expressed in the form

$$
\begin{aligned}
\delta f(\vec{r}, \vec{p}, t) & =\int \vec{\mu}_{0}\left(\vec{r}, \vec{p} ; \vec{r}^{\prime} ; t-t^{\prime}\right) \cdot\left[\delta \vec{F}^{e x t}\left(\vec{r}^{\prime}, t^{\prime}\right)+\int \vec{W}\left(\vec{r}^{\prime}, \vec{r}^{\prime \prime}\right) \delta n\left(\vec{r}^{\prime \prime}, t\right) d \vec{r}^{\prime \prime}\right] d \vec{r}^{\prime} d t^{\prime} \\
& =\int \vec{\mu}_{0}\left(\vec{r}, \vec{p} ; \vec{r}^{\prime} ; t-t^{\prime}\right) \delta \vec{F}^{e f f}\left(\vec{r}^{\prime}, t^{\prime}\right) d \vec{r}^{\prime} d t^{\prime}
\end{aligned}
$$

and in particular denoting $\vec{\nu}_{K S}=\int \vec{\mu}_{0} d \vec{p}$, the density response can be found as

$$
\delta n(r, t) \equiv \int \delta f(\vec{r}, \vec{p}, t) d \vec{p}=\int \vec{\nu}_{K S}\left(\vec{r}, \vec{r}^{\prime}, t-t^{\prime}\right) \cdot \delta \vec{F}^{e f f}\left(\vec{r}^{\prime}, t^{\prime}\right) d \vec{r}^{\prime} d t .
$$

Here $\vec{\nu}_{K S}$ is a classical vector response function giving the density response, to an applied force, of independent electrons moving in the equilibrium force-field $\vec{F}^{(0)}(\vec{r})$.

Since the force in the appropriate gauge is related to a vector potential $a^{\text {eff }}$ by $\delta F^{e f f}=\partial_{t} a^{e f f}, \vec{\nu}_{K S}$ is related to the tensor Kohn-Sham current-current response function $\underline{\chi}_{K S}(\vec{r}, \vec{r}, \omega)[10]$ such that $\vec{j}=\underline{\chi}_{K S} \vec{a}^{e f f}$ by

$$
\vec{\nu}_{K S}=i \omega \vec{\nabla}_{r} \cdot \underline{\chi}_{K S}\left(\vec{r}, \vec{r}^{\prime}, \omega\right)
$$

In obtaining (16) the contiunuity relation $\vec{\nabla} \cdot \vec{j}=i \omega n$ was used. As in the quantal discussion of the ACFDT above, we include in the external force $\vec{F}_{\lambda}^{e x t}$ acting on the $\lambda$-system a sufficient fictitious force, in addition to the true external force, to keep $\vec{F}^{(0)}$ and hence the equilibrium 
density $\lambda$-independent: thus $\vec{F}^{(0)}(\vec{r})$ is the classical equivalent of the gradient of the KS potential.

In the case of homogeneous electron gases, $g_{\lambda}$ is a function only of the separation $R \equiv$ $\left|\vec{r}-\vec{r}^{\prime}\right|$, and then

$$
\frac{\partial}{\partial \vec{R}} \times \vec{W}_{\lambda}=\vec{R} \frac{d g_{\lambda}}{R d R} \times \vec{R} \frac{-\lambda e^{2}}{R^{3}}+g_{\lambda} \frac{\partial}{\partial \vec{R}} \times \frac{\partial}{\partial \vec{R}} \frac{\lambda e^{2}}{R}=\overrightarrow{0}
$$

so that the effective pair force $\vec{W}_{\lambda}$ is irrotational and can be expressed as a gradient of a scalar potential, $\vec{W}_{\lambda}(R)=-(\partial / \partial \vec{R}) w_{\lambda}(R)$. Then, denoting $(\partial / \partial \vec{r}) \cdot \vec{\nu}_{K S}=\chi_{K S}$ (the usual scalar Kohn-Sham density-density response [11], we can use integration by parts (Green's theorem) to write (15) as

$$
\delta n(r, t)=\int \chi_{K S}\left(\vec{r}, \vec{r}^{\prime}, t-t^{\prime}\right) \delta V^{e f f}\left(\vec{r}^{\prime}, t^{\prime}\right) d \vec{r}^{\prime} d t^{\prime}
$$

where

$$
\delta V^{e f f}(\vec{r}, t)=\delta V^{e x t}(\vec{r}, t)+\int w_{\lambda}\left(\left|\vec{r}-\vec{r}^{\prime}\right|\right) \delta n\left(\vec{r}^{\prime}, t\right) d \vec{r}^{\prime} .
$$

and where $\chi_{K S}$ is the (classical) Kohn-Sham density-density response. Since we are dealing with a homogeneous gas, translational invariance allows (17) and (18) to be simplified by space Fourier transformation, giving

$$
\frac{\delta n(\vec{q}, \omega)}{\delta V^{e x t}(\vec{q}, \omega)} \equiv \chi_{\lambda}(q, \omega)=\frac{\chi_{K S}(q, \omega)}{1-w_{\lambda}(q) \chi_{K S}(q, \omega)}
$$

This equation can be recognized as a classical version of the density response of the $\lambda$-system in the Random Phase Approximation (RPA), except that the bare coulomb pair potential $4 \pi \lambda e^{2} / q^{2}$ has been replaced by the effective interaction $w_{\lambda}(q)$. STLS applied this theory to the degenerate electron gas by replacing the classical Boltzmann-equation density response $\chi_{K S}$ by the quantal Lindhard response. At that time, benchmark Diffusion Monte Carlo (DMC) calculations of the uniform gas were not available, but with hindsight one finds a remarkable agreement between STLS and DMC correlation energies, up to values of $r_{s}$ as high as 50 .

In an inhomogeneous system we have, in contrast to the uniform case,

$$
\frac{\partial}{\partial \vec{R}} \times \vec{W}_{\lambda}=\vec{\nabla} g_{\lambda}\left(\vec{r}, \vec{r}^{\prime}\right) \times \frac{\partial}{\partial \vec{r}} \frac{\lambda e^{2}}{\left|\vec{r}-\vec{r}^{\prime}\right|} \neq 0
$$

so that there is no scalar potential corresponding to $\vec{W}_{\lambda}$, and the vector bare response $\vec{\nu}_{K S}$ must be used as in Eq. (15) : the scalar version $\chi_{K S}$ is not sufficient. This is an essential 
difference between the inhomogeneous STLS case as treated in [3] and the homogeneous case. As in the homogeneous case, we postulate that a degenerate Fermi system can be treated via the above semi-classical analysis by using the quantal-Fermi independent-electron response for $\vec{\nu}_{K S}$. By straightforward perturbation of the occupied independent-electron (Kohn-Sham) orbitals $\phi_{j}(\vec{r})$, we obtained for the quantal response at imaginary frequency $i u$

$$
\begin{aligned}
& \vec{\nu}_{K S}\left(\vec{r}, \vec{r}^{\prime}, \omega=i u\right)=\frac{1}{u} 2 \operatorname{Re} \frac{i \hbar e}{m} \sum_{j} f_{j} \phi_{j}^{*}(\vec{r}) \\
& \times\left[G\left(\vec{r}, \vec{r}^{\prime}, E=\hbar \omega_{j}+i \hbar u\right) \vec{\nabla}^{\prime} \phi_{j}\left(\vec{r}^{\prime}\right)-\phi_{j}\left(\vec{r}^{\prime}\right) \vec{\nabla}^{\prime} G\left(\vec{r}, \vec{r}^{\prime}, E=\hbar \omega_{j}+i \hbar u\right)\right]
\end{aligned}
$$

where $f_{j}$ is the Fermi occupation factor and $G$ is the Green function for a single electron moving in the groundstate Kohn-Sham potential $V_{K S}(r)$. G can in general be evaluated as a sum over virtual intermediate states. The Coulomb screening condition (11), (12) for the inhomogeneous case can be written as a Dyson-like "screening" integral equation for the interacting response $\chi_{\lambda}$ :

$$
\begin{aligned}
\chi_{\lambda}\left(\vec{r}, \vec{r}^{\prime}, \omega\right) & =\chi_{K S}\left(\vec{r}, \vec{r}^{\prime}, \omega\right)+\int Q_{\lambda}\left(\vec{r}, \vec{r}^{\prime \prime}, \omega\right) \chi_{\lambda}\left(\vec{r}^{\prime \prime}, \vec{r}^{\prime}, \omega\right) d \vec{r}^{\prime \prime}, \\
Q_{\lambda}\left(\vec{r}, \vec{r}^{\prime \prime}, \omega\right) & =\int \vec{\nu}_{K S}\left(\vec{r}, \vec{r}^{\prime \prime \prime} \omega\right) \cdot \vec{W}_{\lambda}\left(\vec{r}^{\prime \prime \prime}, \vec{r}^{\prime \prime}\right) d \vec{r}^{\prime \prime \prime} .
\end{aligned}
$$

The above scheme was termed [3] the "Inhomogeneous STLS" (ISTLS) approach. It obtained good correlation energies compared to accurate many-body calculations for some simple inhomogeneous systems (i) a helium atom and (ii) charge-neutral jellium slabs with strongly inhomogeneous density profiles. It also provided [13] a smooth transition of the correlation energy of metal slabs as a function of thickness $L$, between known results for the $2 \mathrm{D}$ and $3 \mathrm{D}$ electron gases. It also helped elucidate some problems with the theory of metal surfaces [14].

The same formalism also permits the time-dependent current to be calculated as a momentum moment over the one-body distribution, as follows.

\section{XC KERNEL FROM ISTLS}

In a suitable gauge we can write the effective force on the RHS of (13) in terms of an effective vector potential $\vec{a}^{e f f}$,

$$
\delta \vec{F}^{e f f}=i \omega \vec{a}^{e f f}
$$


Then we can multiply (13) by $m^{-1} \vec{p}$ and integrate over $\vec{p}$ to get the current density perturbation:

$$
\begin{aligned}
\delta j_{\alpha}(\vec{r}, \omega) & =\int \chi_{K S \alpha \beta}\left(\vec{r} ; \vec{r}^{\prime} ; \omega\right) \cdot\left[\vec{a}_{\beta}^{e x t}\left(\vec{r}^{\prime}, \omega\right)+(i \omega)^{-1} \int W_{\beta}\left(\vec{r}^{\prime}, \vec{r}^{\prime \prime}\right) \delta n\left(\vec{r}^{\prime \prime}, \omega\right) d \vec{r}^{\prime \prime}\right] d \vec{r}^{\prime} \\
& =\int \chi_{K S \alpha \beta}\left(\vec{r} ; \vec{r}^{\prime} ; \omega\right) a_{\beta}^{e f f}\left(\vec{r}^{\prime}, \omega\right) .
\end{aligned}
$$

Here

$$
\chi_{K S \alpha \beta}\left(\vec{r} ; \vec{r}^{\prime} ; \omega\right)=i \omega \int m^{-1} p_{\alpha} \mu_{0 \beta}\left(\vec{r}, \vec{p} ; \vec{r}^{\prime} ; \omega\right) d \vec{p} .
$$

is the nonlocal Kohn-Sham current-current response tensor $\underline{\chi}_{K S}$, which in general gives the response of independent (Kohn-Sham) electrons

$$
j_{\alpha}^{\text {indep }}(\vec{r}, \omega)=\int \chi_{K S \alpha \beta}\left(\vec{r} ; \vec{r}^{\prime} ; \omega\right) a_{\beta}^{\text {ext }}\left(\vec{r}^{\prime}, \omega\right) d \vec{r}^{\prime} .
$$

In (23), from (22) we can write the effective vector potential in terms of external part, the standard time-dependent Hartree (RPA) part, and an xc part:

$$
a_{\beta}^{e f f}\left(\vec{r}^{\prime}\right)=a_{\beta}^{e x t}\left(\vec{r}^{\prime}\right)+a_{\beta}^{H}\left(\vec{r}^{\prime}, \omega\right)+a_{\beta}^{x c}\left(\vec{r}^{\prime}, \omega\right)
$$

with

$$
\begin{aligned}
\vec{a}^{H}(\vec{r}, \omega) & =(i \omega)^{-1} \int\left(\vec{\nabla}_{r} \frac{e^{2}}{\left|\vec{r}-\vec{r}^{\prime}\right|}\right) \delta n\left(\vec{r}^{\prime}, \omega\right) d \vec{r}^{\prime} \\
a_{\beta}^{x c}(\vec{r}, \omega) & =(i \omega)^{-1} \int \Delta g\left(\vec{r}, \vec{r}^{\prime}\right)\left(\vec{\nabla}_{r} \frac{e^{2}}{\left|\vec{r}-\vec{r}^{\prime}\right|}\right) \delta n\left(\vec{r}^{\prime}, \omega\right) d \vec{r}^{\prime}, \quad \Delta g=g-1 \\
& =(i \omega)^{-2} \int \Delta g\left(\vec{r}, \vec{r}^{\prime}\right)\left(\vec{\nabla}_{r} \frac{e^{2}}{\left|\vec{r}-\vec{r}^{\prime}\right|}\right) \nabla_{\mu}^{\prime} j_{\mu}\left(\vec{r}^{\prime}, \omega\right) d \vec{r}^{\prime}
\end{aligned}
$$

We used the continuity equation $-i \omega n+\partial_{\mu} j_{\mu}=0$ to relate $\delta n$ to $j$ in the last step. Now in linearized TD current density functional theory [10] the xc vector potential is given by

$$
a_{\beta}^{x c}(\vec{r}, \omega)=\int \mathbf{f}_{\not \beta \mu}^{x c}\left(\vec{r}, \vec{r}^{\prime}, \omega\right) j_{\mu}\left(\vec{r}^{\prime}, \omega\right) d \vec{r}^{\prime}
$$

and so we can use (28) to identify the tensor xc kernel of ISTLS theory, in the ACFDT sense:

$$
\mathbf{f}_{\beta \mu}^{x c, I S T L S}\left(\vec{r}, \vec{r}^{\prime}\right)=\frac{1}{\omega^{2}}\left(g\left(\vec{r}, \vec{r}^{\prime}\right)-1\right) \partial_{\beta}\left(\frac{e^{2}}{\left|\vec{r}-\vec{r}^{\prime \prime}\right|}\right) \partial_{\mu}^{\prime} .
$$

Eq (30) is a principal result of the present work. 
Note that the gradient $\partial_{\beta}$ does not operate on the entire expression (30). Despite this unsymmetric appearance, (30) does conserve total momentum, in the sense that the total xc self-force on the entire electron gas is zero. This follows because the linear perturbation to the total xc self-force force on the entire inhomogeneous electron gas is

$$
\delta \vec{F}^{t o t}=\int\left(\vec{F}^{0 x c}(\vec{r}) \delta n(\vec{r})+\delta \vec{F}^{x c}(\vec{r}) n_{0}(\vec{r})\right) d \vec{r}
$$

Using (10), (11) and (12) we can write this as

$$
\begin{aligned}
\delta \vec{F}^{t o t} & =\int\left(\left(\int \Delta g\left(\vec{r}, \vec{r}^{\prime}\right) \vec{\nabla}_{r} \frac{e^{2}}{\left|\vec{r}-\vec{r}^{\prime}\right|} n_{0}\left(\vec{r}^{\prime}\right) d \vec{r}^{\prime}\right) \delta n(\vec{r})\right. \\
& \left.\left.+\iint \Delta g\left(\vec{r}, \vec{r}^{\prime}\right) \vec{\nabla}_{r} \frac{e^{2}}{\left|\vec{r}-\vec{r}^{\prime}\right|} \delta n\left(\vec{r}^{\prime}\right) d \vec{r}^{\prime}\right) n_{0}(\vec{r})\right) d \vec{r} \\
& =\iint \Delta g\left(\vec{r}, \vec{r}^{\prime}\right)\left(\delta n(\vec{r}) n_{0}\left(\vec{r}^{\prime}\right)+n_{0}(\vec{r}) \delta n\left(\vec{r}^{\prime}\right)\right) \vec{\nabla}_{r} \frac{e^{2}}{\left|\vec{r}-\vec{r}^{\prime}\right|} d \vec{r} d \vec{r}^{\prime}=\overrightarrow{0}
\end{aligned}
$$

where $\Delta g=g-1$. The zero on the RHS arises because $\vec{\nabla}_{r} \frac{e^{2}}{\left|\vec{r}-\vec{r}^{\prime}\right|}$ is antisymmetric under interchange of $\vec{r}$ and $\vec{r}^{\prime}$, whereas the other factor $\Delta g\left(\vec{r}, \vec{r}^{\prime}\right)\left(\delta n(\vec{r}) n_{0}\left(\vec{r}^{\prime}\right)+n_{0}(\vec{r}) \delta n\left(\vec{r}^{\prime}\right)\right)$ is symmetric. It is possible that the unsymmetric appearance of (30) is related to violation of dynamic hole normalization as noted in (2). However, simply making the $\partial_{\beta}$ operator act on the whole right-hand side in (30), while restoring a symmetric appearance, could cause violation of momentum conservation unless the groundstate terms are also modified.

\section{RELATION TO THE PGG KERNEL}

In the ISTLS formalism, $g\left(\vec{r}, \vec{r}^{\prime}\right)$ in $(30)$ is the groundstate spatial correlating factor determined selfconsistently from the response $\chi$ corresponding to (20) and (21) via the Fluctuation Dissipation Theorem. However, we could make simpler choices for $g$. For example, we still obtain a non-trivial theory if we choose the Hartree Fock correlating factor based on the occupied groundstate KS orbitals,

$$
\begin{aligned}
\Delta g & =g^{H F}\left(\vec{r}, \vec{r}^{\prime}\right)-1 \equiv \frac{n_{2}^{H F}}{n(\vec{r}) n\left(\vec{r}^{\prime}\right)}-1 \\
& =\frac{1}{n(\vec{r}) n\left(\vec{r}^{\prime}\right)} \sum_{m, n o c c} \phi_{m}^{*}(\vec{r}) \phi_{n}^{*}\left(\vec{r}^{\prime}\right)\left[\phi_{m}(\vec{r}) \phi_{n}\left(\vec{r}^{\prime}\right)-\phi_{n}(\vec{r}) \phi_{m}\left(\vec{r}^{\prime}\right)\right]-1 \\
& =\frac{-1}{n(\vec{r}) n\left(\vec{r}^{\prime}\right)} \sum_{m, n o c c} \phi_{m}^{*}(\vec{r}) \phi_{n}^{*}\left(\vec{r}^{\prime}\right) \phi_{n}(\vec{r}) \phi_{m}\left(\vec{r}^{\prime}\right)=\frac{-1}{\left.n(\vec{r}) n\left(\vec{r}^{\prime}\right)\right)}\left|\sum_{m o c c} \phi_{m}^{*}(\vec{r}) \phi_{m}\left(\vec{r}^{\prime}\right)\right|^{2}
\end{aligned}
$$


Then (30) becomes

$$
\mathbf{f}_{\beta \mu}^{x}\left(\vec{r}, \vec{r}^{\prime}\right)=\frac{1}{\omega^{2}} \frac{-1}{\left.n(\vec{r}) n\left(\vec{r}^{\prime}\right)\right)}\left|\sum_{m o c c} \phi_{m}^{*}(\vec{r}) \phi_{m}\left(\vec{r}^{\prime}\right)\right|^{2} \partial_{\beta} \frac{e^{2}}{\left|\vec{r}-\vec{r}^{\prime}\right|} \partial_{\mu}^{\prime}
$$

This is clearly closely related to the approximate TDOEP (time-dependent optimized effective potential) scheme of Petersilke, Gossmann and Gross ([15, 16],"PGG"). These authors gave the scalar xc kernel of TD density functional theory as

$$
f^{P G G}\left(\vec{r}, \vec{r}^{\prime}\right)=\frac{-1}{n(\vec{r}) n\left(\vec{r}^{\prime}\right)} \frac{e^{2}}{\left|\vec{r}-\vec{r}^{\prime}\right|}\left|\sum_{m o c c} \phi_{m}^{*}(\vec{r}) \phi_{m}\left(\vec{r}^{\prime}\right)\right|^{2}
$$

Thus we can regard the ISTLS scheme for $\mathbf{f}_{x c, \beta \mu}^{I S T L S}\left(\vec{r}^{\prime}, \vec{r}^{\prime}\right)($ Eq. (30)) as a generalization of the PGG scheme, apart from the positioning of the $\partial_{\beta}$ operator in (32) and (30).

\section{RELATION TO THE VIGNALE-KOHN-ULLRICH-CONTI LINEAR RE- SPONSE FORMALISM}

Vignale and Kohn [10] derived a local approximation for the tensor exchange-correlation kernel $\mathbf{f}_{x c, \beta \mu}$ of a weakly inhomogeneous electron gas, valid to second order in the spatial gradients, where $\mathbf{f}_{x c, \beta \mu}$ is defined in general by (29). This formalism was extended and simplified by Vignale, Ullrich and Conti (VUC, [17]).

The Vignale-Kohn $\mathbf{f}_{\mathbf{x c}}$ is local, i.e. contains $\delta\left(\vec{r}-\vec{r}^{\prime}\right)$ and its space derivatives. At first sight (30) appears to represent a nonlocal generalization of just the first of many terms in the VK xc kernel (Eq (19) of [10]), namely

$$
\mathbf{f}_{x c, \beta \mu}^{V K, 0}\left(\vec{r}, \vec{r}^{\prime}\right)=\frac{1}{\omega^{2}} \delta\left(\vec{r}-\vec{r}^{\prime}\right) \partial_{\beta} f_{x c L}^{h}\left(n_{0}(\vec{r}), \omega\right) \partial_{\mu}^{\prime}
$$

where $f_{x c L}^{h}(\omega)$ is the longitudinal xc kernel of the homogeneous gas. All the other terms in the VK expression either vanish when $f_{x c, L}$ is taken to be frequency-independent (i.e. when $\delta f_{x c L}^{h} \equiv f_{x c L}^{h}(\omega)-f_{x c L}^{h}(0)=0$ in the notation of [10]), or else they involve the transverse part $f_{x c T}^{h}(\omega)$ of the homogenous xc kernel, related e.g. to shear viscosity. Thus ISTLS can perhaps be viewed as a static but nonlocal modification of the longitudinal viscosity/pressure effects only. As such it will correspond best with the VK [10] and VUC [17] formalisms for the case where spatial variation of all quantities is restricted to a single cartesian direction. 


\section{PROSPECTS FOR INTRODUCING A VELOCITY DEPENDENCE}

In order to describe better the physics of shear flows one might consider applying the STLS ideas in a momentum-resolved fashion, e.g.

$$
f_{\lambda}^{(2)}\left(\vec{r}, \vec{p} ; \vec{r}^{\prime}, \vec{p}^{\prime}, t\right) \approx \Gamma_{\lambda}\left(\vec{r}, \vec{p}: \vec{r}^{\prime} \vec{p}^{\prime}\right) f(\vec{r}, \vec{p}, t) f\left(\vec{r}^{\prime}, \vec{p}^{\prime}, t\right)
$$

where $\Gamma$ is derived from the equilibrium pair and 1-body distributions $f_{0}^{(2)}$ and $f_{0}$ :

$$
\Gamma_{\lambda}\left(\vec{r}, \vec{p}: \vec{r}^{\prime} \vec{p}^{\prime}\right)=\frac{f_{0}^{(2)}\left(\left(\vec{r}, \vec{p} ; \vec{r}^{\prime}, \vec{p}^{\prime}\right)\right.}{f_{0}(\vec{r}, \vec{p}) f_{0}\left(\vec{r}^{\prime}, \vec{p}^{\prime}\right)}
$$

Of course (33) will presumably, like the regular STLS Ansatz (1), violate dynamic hole normalization as in (2). Let us assume that this normalization condition is unimportant, or that it can be corrected. Then the essential point is that the fluctuation-dissipation theorem can be used to obtain $f_{0}^{(2)}\left(\vec{r}, \vec{p} ; \vec{r}^{\prime}, \vec{p}^{\prime}\right)$ from the linear response $\chi\left(\vec{r}, \vec{p} ; \vec{r}^{\prime}, \vec{p}^{\prime}, \omega\right)$ of the 1-body distribution $\delta f(\vec{r}, \vec{p}, \omega)$ to a momentum-dependent perturbation acting at the point $\left(\vec{r}^{\prime}, \vec{p}^{\prime}, \omega\right)$. This response is available from the linearized form of the kinetic equation (8) in which the right side is closed by use of (33), thus creating a selfconsistency loop as in the left side of Fig. 1, but now with momentum resolution as well as spatial resolution. The dynamic xc stress tensor [17] can be obtained as the second momentum moment of the ISTLS dynamic distribution $f(\vec{r}, \vec{p}, t)$ This will have to be done carefully, and constraints such as the vanishing of the total xc self-force (momentum conservation), and the satisfaction of the Acceleration Theorem and hence the Harmonic Potential / generalized Kohn Theorem [18-20] will have to be checked if a frequency dependence is also present as in the following section. It is interesting to speculate whether the tensor behaviour of the resulting xc kernel will have the full richness of the Vignale-Kohn [10] or Vignale-Ullrich-Conti [17] kernels that apply for systems with slow spatial variation of the groundstate density.

\section{PROSPECTS FOR INTRODUCING A FREQUENCY DEPENDENCE}

The only frequency dependence of the ISTLSkernel $\mathbf{f}_{x c}$ of Eq. (30) is the trivial $\omega^{-2}$ factor corresponding to conversion between currents and densities via the continuity equation $i \omega n=\vec{\nabla} \cdot \vec{j}$, and between forces and vector potentials via $\vec{F}=i \omega \vec{a}$. The interest here is in producing a nontrivial frequency dependence along with the ISTLS type of spatial 
nonlocality exhibited by (30). This is a difficult problem whose solution is not immediately obvious.

The frequency dependence of the VK type of kernel is equivalent to a memory effect in the relation between effective vector potential $\vec{a}^{e f f}$ and current $\vec{j}$. How might such a memory be obtained in the language of pair functions that underlies the STLS philosophy? One possible answer comes from linearizing the basic STLS factorization assumption (1)

$$
f_{\lambda}^{(2)}\left(\vec{r}, \vec{p} ; \vec{r}^{\prime}, \vec{p}^{\prime}, t\right) \approx g_{\lambda}\left(\vec{r}, \vec{r}^{\prime}\right) f(\vec{r}, \vec{p}, t) f\left(\vec{r}^{\prime}, \vec{p}^{\prime}, t\right)
$$

giving

$$
\delta f_{\lambda}^{(2)}\left(\vec{r}, \vec{p} ; \vec{r}^{\prime}, \vec{p}^{\prime}, t\right) \approx g_{\lambda}\left(\vec{r}, \vec{r}^{\prime}\right)\left(f_{0}(\vec{r}, \vec{p}) \delta f\left(\vec{r}^{\prime}, \vec{p}^{\prime}, t\right)+\delta f(\vec{r}, \vec{p}, t) f_{0}\left(\vec{r}^{\prime}, \vec{p}^{\prime}\right)\right)
$$

In order to introduce memory we consider replacing this by

$$
\delta f_{\lambda}^{(2)}\left(\vec{r}, \vec{p} ; \vec{r}^{\prime}, \vec{p}^{\prime}, t\right) \approx \int_{0}^{\infty} \gamma_{\lambda}\left(\vec{r}, \vec{r}^{\prime} \tau\right)\left(f_{0}(\vec{r}, \vec{p}) \delta f\left(\vec{r}^{\prime}, \vec{p}^{\prime}, t-\tau\right)+\delta f(\vec{r}, \vec{p}, t-\tau) f_{0}\left(\vec{r}^{\prime}, \vec{p}^{\prime}\right)\right)
$$

It would be tempting to assume that $\gamma$ can be obtained solely from consistency with the fluctuation-dissipation theorem as in the usual STLS/ISTLS approach. After all, the FDT does not simply give the equal-time pair correlations in terms of the frequency integrals of response functions: it also allows prediction of correlation functions at a specific frequency $\omega$, or eqivalently the dynamic pair distribution $f^{e q u}\left(r p, t ; r^{\prime} p^{\prime} t^{\prime}\right)$ with $t \neq t^{\prime}$. However inspection of (8) reveals that the kinetic theory approach does not use the unequal-time dynamic pair distribution: rather we have postulated that it is the relation (in the noneqilibrium situation) between the (equal-time) pair distribution $f_{2}$ and the one-distribution $f$ that is time-delayed as in (35). Thus it seems that STLS-like self-consistency via the fluctuationdissipation theorem will probably not be enough, on its own, to determine the required frequency dependence. Perhaps some guidance can be obtained by extrapolating the theory to a nearly-uniform density and using the known Vignale-Kohn-Ullrich-Conti [10, 17] results, valid in the slowly-varying limit, to determine the time delay properties.

\section{SUMMARY}

We have shown that the inhomogeneous STLS (ISTLS, ) scheme [3] can be associated with a nonlocal tensor exchange-correlation kernel in the context of linear Time Dependent 
Current Density Functional Theory. This expression however has only a relatively trivial tensorial character and frequency dependence. Nevertheless the attractive feature of ISTLS is that it generates the xc kernel self-consistently for the particular system under study, even for highly inhomogeneous systems, without appeal to properties of the homogeneous electron gas. In this preliminary survey we have suggested ways in which this feature might be retained while incorporating more of the known physics. This could include shear viscosity and memory effects, which for systems with slow spatial inhomogeneity are described by the Vignale-Kohn-Ullrich-Conti formalism of TDCDFT [10, 17].

[1] K. S. Singwi, M. P. Tosi, R. H. Land, and A. Sjolander, Phys. Rev. 176, 589 (1968).

[2] T. Hasegawa and M. Shimizu, J. Phys. Soc. Jap. 38, 965 (1975).

[3] J. F. Dobson, J. Wang, and T. Gould, Phys. Rev. B 66, 081108(R) (2002).

[4] J. F. Dobson and B. P. Dinte, Phys. Rev. Lett. 76, 1780 (1996).

[5] D. M. Ceperley and B. J. Alder, Phys. Rev. Lett. 45, 566 (1980).

[6] H. Hayashi and M. Shimizu, J. Phys. Soc. Jap. 48, 16 (1980).

[7] K. S. Singwi, A. Sjölander, M. P. Tosi, and R. H. Land, Phys. Rev. B 1, 1044 (1970).

[8] A. Holas and S. Rahman, Phys. Rev. B 35, 2720 (1987).

[9] P. Vashishta and K. S. Singwi, Phys. Rev. B 6, 875 (1972).

[10] G. Vignale and W. Kohn, Phys. Rev. Lett. 77, 2037 (1996).

[11] E. K. U. Gross and W. Kohn, Phys. Rev. Lett. 55, 2850 (1985).

[12] T. J. M. Boyd and J. J. Sanderson, Plasma Dynnamics (Barnes and Noble, New York, 1969).

[13] L. A. Constantin, J. P. Perdew, and J. M. Pitarke, Phys. Rev. Lett. 101, 016406 (2008).

[14] L. A. Constantin, J. M.Pitarke, J. F. Dobson, A. Garcia-Lekue, and J. P. Perdew, Phys. Rev. Lett. 100, 036401 (2008).

[15] M. Petersilka, U. J. Gossmann, and E. K. U. Gross, Phys. Rev. Lett. 76, 1212 (1996).

[16] M. Petersilka, U. J. Gossmann, and E. K. U. Gross, in Electronic Density Functional Theory, edited by J. F. Dobson, G. Vignale, and M. P. Das (Plenum, New York, 1998), Chap. 13.

[17] G. Vignale, C. A. Ullrich, and S. Conti, Phys. Rev. Lett. 79, 4878 (1997).

[18] G. Vignale, Phys. Letts. A 209, 206 (1995).

[19] J. F. Dobson, Phys. Rev. Lett. 73, 2244 (1994). 
[20] L. Brey et al., Phys. Rev. B 40, 10647 (1989).

\section{APPENDIX: QUANTITIES USED IN THIS WORK FROM A MANY-BODY WAVEFUNCTION PERSPECTIVE}

The density-density response function $\chi\left(\vec{r}, \vec{r}^{\prime}, t-t^{\prime}\right)$ is defined as the linear response of the electron density at space-time point $\vec{r}, t$ to a perturbation in the applied oneelectron scalar potential energy function $V^{e x t}$ at point $\vec{r}^{\prime}, t^{\prime}$. Assuming a perturbation of form $\delta V^{e x t}\left(\vec{r}^{\prime}\right) \exp (-i \omega t)$ so that the density pertubation $\delta n(\vec{r}, t)$ is of form $\delta n(\vec{r}) \exp (-i \omega t)$ by time translational invariance, we can then define the time-Fourier transformed response function $\chi$ by

$$
\delta n(\vec{r})=\int \chi\left(\vec{r}, \vec{r}^{\prime}, \omega\right) \delta V^{e x t}\left(\vec{r}^{\prime}\right) d^{3} \vec{r}^{\prime}
$$

From first order perturbation theory one can obtain the following exact expression for $\chi$ :

$$
\chi\left(\vec{r}, \vec{r}^{\prime}, \omega=i u\right)=-\sum_{J} \frac{\langle 0|\delta \hat{n}(\vec{r})| J\rangle\left\langle J\left|\delta \hat{n}\left(\vec{r}^{\prime}\right)\right| 0\right\rangle}{E_{0}-E_{J}-i \hbar u}
$$

where $|0\rangle$ is the groundstate, and $\left\langle\vec{r}_{1}, \vec{r}_{2}, \ldots, \vec{r}_{N} \mid 0\right\rangle=\Psi_{0}\left(\vec{r}_{1}, \vec{r}_{2}, \ldots, \vec{r}_{N}\right)$ is the groundstate many body wavefunction. $\delta \hat{n}(\vec{r})$ is the density fluctuation operator at point $\vec{r}$, defined by

$$
\delta \hat{n}(\vec{r})=\sum_{a} \delta^{3}\left(\vec{r}-\hat{\mathbf{r}}_{a}\right)-n(\vec{r})
$$

where $\hat{\mathbf{r}}_{a}$ is the position operator for the electron labelled $a$ and $n(\vec{r})$ is the groundstate electron number density defined in the usual way:

$$
n(\vec{r})=\langle\hat{n}(\vec{r})\rangle=\left\langle\sum_{a} \delta^{3}\left(\vec{r}-\hat{\mathbf{r}}_{a}\right)\right\rangle_{0}=N \int\left|\Psi_{0}\left(\vec{r}, \vec{r}_{2}, \vec{r}_{3}, \ldots \vec{r}_{N}\right)\right|^{2} d^{3} r_{2} d^{3} r_{3} \ldots d^{3} r_{N}
$$

Here appropriate normalization and correct particle-exchange antisymmetry have been assumed for the many-electron wavefunction $\Psi_{0}$. 